\title{
Geometric actions for three-dimensional gravity
}

\author{
G. Barnich ${ }^{1}$ H. A. González ${ }^{2}$ and P. Salgado-Rebolledo ${ }^{3}$ \\ ${ }^{1}$ Physique Théorique et Mathématique \\ Université Libre de Bruxelles and International Solvay Institutes \\ Campus Plaine C.P. 231, B-1050 Bruxelles, Belgium \\ ${ }^{2}$ Institute for Theoretical Physics \\ Vienna University of Technology \\ Wiedner Hauptstr. 8-10/136, A-1040, Vienna, Austria \\ ${ }^{3}$ Facultad de Ingeniería y Ciencias and UAI Physics Center \\ Universidad Adolfo Ibañez \\ Avda. Diagonal las Torres 2640, Peñalolén, Santiago, Chile
}

\begin{abstract}
The solution space of three-dimensional asymptotically antide Sitter or flat Einstein gravity is given by the coadjoint representation of two copies of the Virasoro group in the former and the centrally extended $\mathrm{BMS}_{3}$ group in the latter case. Dynamical actions that control these solution spaces are usually constructed by starting from the Chern-Simons formulation and imposing all boundary conditions. In this note, an alternative route is followed. We study in detail how to derive these actions from a group-theoretical viewpoint by constructing geometric actions for each of the coadjoint orbits, including the appropriate Hamiltonians. We briefly sketch relevant generalizations and potential applications beyond three-dimensional gravity.
\end{abstract}




\section{Contents}

1 Introduction 3

2 Review of geometric actions

2.1 Kinetic term . . . . . . . . . . . . . . . . . . 5

2.2 Symmetric Hamiltonians and deformations . . . . . . . . . . . . 7

3 Geometric actions for centrally extended groups 9

3.1 Central extensions . . . . . . . . . . . . . . . . . . . . . 9

3.2 Geometric actions for central extensions . . . . . . . . . . . . . . 11

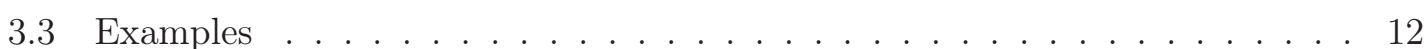

3.3 .1 Kac-Moody groups . . . . . . . . . . . . . . . . . 12

3.3 .2 Virasoro Group . . . . . . . . . . . . . . . . . . . . . . . . 14

4 Geometric action for semi-direct products $\quad 16$

4.1 Semi-direct product groups . . . . . . . . . . . . . . . 16

4.2 Geometric actions for $\mathcal{S}_{\mathrm{Ad}} \ldots \ldots \ldots \ldots \ldots \ldots \ldots$

4.3 Geometric action for centrally extended $\widehat{\mathcal{S}}_{\text {Ad }} \ldots \ldots \ldots \ldots$

4.4 Examples . . . . . . . . . . . . . . . . . . . . . . . . 19

4.4.1 Loop groop of $G \ltimes \mathfrak{g}$ and its extension . . . . . . . . . . 19

$4.4 .2 \widehat{\mathrm{BMS}}_{3} \operatorname{group} \ldots \ldots \ldots \ldots \ldots \ldots$

5 Discussion and perspectives

\begin{tabular}{ll} 
Acknowledgments & 24 \\
\hline
\end{tabular} 


\section{Introduction}

Even though three-dimensional general relativity does not admit local degrees of freedom, there is both an infinite dimensional symmetry structure [1] and rich dynamics in two dimensions [2] when allowing for non-trivial boundary conditions. The standard "bulk" approach to derive this dynamics starts from the Chern-Simons formulation [3,4]: when taking into account the boundary conditions and the associated surface terms [5], this leads one in a first step to a Wess-Zumino-Witten theory along the lines of [6 8], and in a second step to Liouville theory through a Hamiltonian reduction [9 11]. In this approach, the Hamiltonian of the dual theory is inherited from the surface term associated with time translations. Alternatively, the dual theory may be contructed through holographic renormalization in the context of the $\mathrm{AdS}_{3} / \mathrm{CFT}_{2}$ correspondence [12].

In this paper, we follow a different method to construct dual two-dimensional action principles for the gauge fixed solution spaces of three-dimensional gravity. Indeed, both in the asymptotically AdS and flat cases, the solution space coincides with the centrally extended coadjoint representation, at fixed values of the central charges, of the asymptotic symmetry groups, viz. two copies of the Virasoro group [1, 12 17] and the centrally extended $\widehat{\mathrm{BMS}}_{3}$ group [18 24], respectively (see also [25] 29] for recent related considerations). More precisely, the general solution to the Einstein equations with AdS asymptotics is given by

$$
d s^{2}=\frac{l^{2}}{r^{2}} d r^{2}-\left(r d x^{+}-\frac{8 \pi G l}{r} b^{-} d x^{-}\right)\left(r d x^{-}-\frac{8 \pi G l}{r} b^{+} d x^{+}\right),
$$

with $x^{ \pm}=\frac{t}{l} \pm \varphi$ and the arbitrary $2 \pi$-periodic functions $b^{ \pm}\left(x^{ \pm}\right)$transforming as

$$
\tilde{b}^{ \pm}=\left(\partial_{ \pm} f^{ \pm}\right)^{2} b^{ \pm} \circ f^{ \pm}-c^{ \pm} S_{x^{ \pm}}\left[f^{ \pm}\right], \quad c^{ \pm}=\frac{3 l}{2 G},
$$

under the conformal transformations $x^{ \pm} \rightarrow f^{ \pm}\left(x^{ \pm}\right), f^{ \pm}\left(x^{ \pm}+2 \pi\right)=f^{ \pm}\left(x^{ \pm}\right) \pm 2 \pi$ of the cylinder at infinity, with the Schwarzian derivative given by

$$
S_{x}[f]=\frac{1}{24 \pi}\left[\partial_{x}^{2}\left(\log \partial_{x} f\right)-\frac{1}{2}\left(\partial_{x}\left(\log \partial_{x} f\right)\right)^{2}\right] .
$$

This coincides with the coadjoint representation of the two copies of the Virasoro group for fixed values of the central charges. For asymptotically flat spacetimes, one finds instead

$$
d s^{2}=2\left[8 \pi G p d u-d r+8 \pi G\left(j+u p^{\prime}\right) d \varphi\right] d u+r^{2} d \varphi^{2},
$$

where the arbitrary $2 \pi$-periodic functions $p=p(\varphi), j=j(\varphi)$ transform as

$$
\begin{array}{r}
\tilde{p}=\left(f^{\prime}\right)^{2} p \circ f-c_{2} S_{\varphi}[f], \quad c_{2}=\frac{3}{G}, \\
\tilde{j}=\left(f^{\prime}\right)^{2}\left[j+\alpha p^{\prime}+2 \alpha^{\prime} p-\frac{c_{2}}{24 \pi} \alpha^{\prime \prime \prime}\right] \circ f-c_{1} S_{\varphi}[f], \quad c_{1}=0,
\end{array}
$$


under the $\mathrm{BMS}_{3}$ transformations $(u, \varphi) \rightarrow\left(f^{\prime} u+\alpha(f(\varphi)), f(\varphi)\right)$, with $f(\varphi+2 \pi)=$ $f(\varphi)+2 \pi$ and $\alpha(\varphi+2 \pi)=\alpha(\varphi)$. In turn, this coincides wih the coadjoint representation of the centrally extended $\mathrm{BMS}_{3}$ group for fixed values of the central charges.

As a consequence, the gravitational solution space admits a partition into coadjoint orbits. For any group $G$, the individual orbits are homogeneous symplectic spaces $G / \mathcal{H}$ (see e.g. 30 33 and original references therein). The aspect we will exploit here is that one can associate to each of these orbits, in a canonical way, geometric actions which admit $G$ as a global and $\mathcal{H}$ as a gauge symmetry group [34. What is fixed in these actions is the kinetic term determined by the symplectic structure on the coadjoint orbit. If the aim is to construct $G$-invariant dynamical systems, one may choose a suitable Hamiltonian defined on the coadjoint orbit that respects $G$-invariance (see also e.g. [35, 36] for reviews).

When this method is applied to three-dimensional gravity, we will get finer actions than those of [2,22, 37, 38], precisely adapted to the individual orbits. From the bulk point of view, they take additional information on non-trivial holonomies into account. In the anti-de Sitter case for example, one finds an intriguing connection between $3 d$ and $2 d$ gravity in the sense that the geometric actions for each copy of the Virasoro group differ from the action for two-dimensional gravity by taking into account a more general covector, for which not only the central charge differs from zero [11, 39 42]. In the flat case, this approach allows us to construct novel $\widehat{\mathrm{BMS}}_{3}$ invariant actions (see also [43]). Note that the Hamiltonian can be fixed by reverting to the bulk approach described above in order to identify a suitable generator.

Another interesting aspect of this approach to two-dimensional conformal or $\mathrm{BMS}_{3}$ invariant actions is that, exactly like in the case of loop groups and the associated Wess-Zumino-Witten theories, they can also be interpreted as one-dimensional particle/world-line actions associated to infinite-dimensional groups. The spatial dimension is hidden or emergent, depending on whether one uses a Fourier expansion for the Lie algebra generators and their duals with associated infinite mode sums or an inner product with an explicit integration over the circle. For instance, from the two-dimensional point of view, the deformation by a Wess-Zumino-Witten term involves a 3-cocycle of the Lie algebra of the group $G$, whereas in the worldline approach, this deformation comes from a related 2-cocycle on the Lie algebra of the loop group of $G$.

The structure of the paper is as follows: in the next section, we review the construction of geometric actions, with a special emphasis on how to include Hamiltonians that preserve $G$-invariance. We continue with a discussion of central extensions and the well-known examples of the Kac-Moody and Virasoro group relevant for three-dimensional asymptotically anti de-Sitter gravity. We then move to semi- 
direct product groups in order to be able to treat three-dimensional flat gravity. In this section, we provide novel $\mathfrak{i s o}(2,1)$ WZW models and $\mathrm{BMS}_{3}$ invariant field theories in two dimensions. In the last section, we relate our considerations to recent developments in the field and discuss future prospects, both for three and four dimensional gravity.

\section{Review of geometric actions}

\subsection{Kinetic term}

The adjoint action of a Lie group $G$ on its Lie algebra $\mathfrak{g}$ is defined as the differential of the automorphism $h \mapsto g h g^{-1}$ at the identity

$$
\operatorname{Ad}_{g} X=\left.\frac{d}{d s}\left(g h(s) g^{-1}\right)\right|_{s=0},
$$

where $X=\left.\frac{d h(s)}{d s}\right|_{s=0} \in \mathfrak{g}$. The coadjoint action of $G$ on the dual space $\mathfrak{g}^{*}$ of $\mathfrak{g}$ is defined as

$$
\left\langle\operatorname{Ad}_{g^{-1}}^{*} b, X\right\rangle=\left\langle b, \operatorname{Ad}_{g} X\right\rangle,
$$

where $b \in \mathfrak{g}^{*}$ and $\langle\cdot, \cdot\rangle$ is the pairing between $\mathfrak{g}$ and $\mathfrak{g}^{*}$. For a fixed element $b_{0}$ of $\mathfrak{g}^{*}$, this action generates a coadjoint orbit $O_{b_{0}}$, the set of elements $b \in \mathfrak{g}^{*}$ such that

$$
b=\mathrm{Ad}_{g^{-1}}^{*} b_{0},
$$

which is a manifold isomorphic to $G / \mathcal{H}_{b_{0}}$, with $\mathcal{H}_{b_{0}}$ the isotropy group of $b_{0}$ under the coadjoint action, i.e., the subgroup of elements $h \in G$ satisfying $\operatorname{Ad}_{h}^{*} b_{0}=b_{0}$.

Coadjoint orbits are particulary interesting as they are symplectic manifolds. The Kirillov-Kostant symplectic form is the pull-back to a coadjoint orbit $O_{b_{0}}$ of the pre-symplectic form on $G$ given by

$$
\Omega=\frac{1}{2}\left\langle b, \operatorname{ad}_{\theta} \theta\right\rangle,
$$

where $b=\operatorname{Ad}_{g^{-1}}^{*} b_{0}$ is a point on the orbit, $\theta$ is the left invariant Maurer-Cartan form satisfying

$$
d \theta=-\frac{1}{2} \operatorname{ad}_{\theta} \theta
$$

and ad denotes the adjoint action of $\mathfrak{g}$ on itself. As $\Omega$ is closed, it is locally exact. In fact,

$$
\Omega=d a, \quad a=\langle b, \theta\rangle,
$$

and therefore, a geometric action $I_{\mathrm{G}}\left[g ; b_{0}\right]$ can be defined on the phase space through

$$
I_{\mathrm{G}}\left[g ; b_{0}\right]=\int_{\gamma} a,
$$


where $\gamma$ is a path on the coadjoint orbit $O_{b_{0}}$.

In particular, for finite dimensional matrix groups, a local solution to (2.5) is simply $\theta=g^{-1} d g$ and the pre-symplectic potential becomes $a=\left\langle b_{0}, d g g^{-1}\right\rangle$. The first order Euler-Lagrange equations of motion are equivalent to setting to zero the one-forms $i_{V} \Omega$ where $V$ is the vector field associated to $\frac{d g}{d t}$ and $t$ parametrizes the path $\gamma$. Note also that one may (trivially) write this action in Wess-Zumino-Witten form,

$$
I_{\mathrm{G}}\left[g ; b_{0}\right]=\int_{\Sigma} \Omega
$$

The assumptions here are that fields are extended to $\Sigma$ and that $\gamma$ is part of the boundary of $\Sigma$, with suitable conditions on fields and their derivatives such that all other boundary terms vanish.

Consider the vector fields associated to one parameter families of right translations generated by $X, v_{X}^{R}=\left.\frac{d}{d s}\left(g h_{R}(s)\right)\right|_{s=0}$ with $\left.\frac{d}{d s} h_{R}(s)\right|_{s=0}=X$. These vector fields are the left invariant vector fields that reduce to $X$ at the identity. For all $X \in \mathfrak{g}$ that are constant along the path, they define global symmetries of $I_{\mathrm{G}}\left[g ; b_{0}\right]$. This follows from

$$
i_{v_{X}^{R}} \Omega=d Q_{X}, \quad Q_{X}=-\langle b, X\rangle,
$$

and $£_{v_{X}^{R}} a=i_{v_{X}^{R}} \Omega+d\langle b, X\rangle$. The associated Noether charge is $Q_{X}$.

Elements $\epsilon$ of $\mathfrak{h}_{b_{0}}$, the "little" algebra associated to $\mathcal{H}_{b_{0}}$, are defined by $\operatorname{ad}_{\epsilon}^{*} b_{0}=0$. Let $v_{\epsilon}^{L}$ be the vector fields associated to one parameter families of left translations by elements $h_{L}(s)$ of $\mathcal{H}_{b_{0}}$ and generated by $\epsilon, v_{\epsilon}^{L}=\left.\frac{d}{d s}\left(h_{L}(s) g\right)\right|_{s=0}$ with $\left.\frac{d}{d s} h_{L}(s)\right|_{s=0}=\epsilon$. These vector fields are the right invariant vector fields that reduce to $\epsilon$ at the identity. Let us now assume furthermore that $\epsilon=\epsilon(t)$ depends a priori arbitrarily on $t$. It then follows from $£_{v_{\epsilon}^{L}} a=i_{v_{\epsilon}^{L}} \Omega+d\left\langle b_{0}, \epsilon\right\rangle$ and

$$
i_{v_{\epsilon}^{L}} \Omega=0
$$

that these transformations define gauge symmetries. More precisely, the action $I_{\mathrm{G}}\left[g ; b_{0}\right]$ is gauge invariant provided that $\epsilon(t)$ vanishes at the end points of $\gamma$. Note that $Q_{X}$ is gauge invariant since

$$
£_{v_{\epsilon}^{L}} Q_{X}=0,
$$

by using (2.9) and (2.10). Note also that the Noether charges form a representation under the action of the global symmetries,

$$
£_{v_{X_{1}}^{R}} Q_{X_{2}}=Q_{\left[X_{1}, X_{2}\right]} .
$$




\subsection{Symmetric Hamiltonians and deformations}

One can always add to the geometric action (2.7) a gauge invariant function on the orbit playing the role of a Hamiltonian. The inclusion of such a Hamiltonian generically breaks some of the global symmetries of $I_{\mathrm{G}}\left[g ; b_{0}\right]$. In the spirit of effective theory, one is interested in Hamiltonians or other deformations that preserve global symmetries.

(i) One possibility that preserves all global symmetries is to consider an extended action, where one of the Noether charges plays the role of the Hamiltonian, $H_{X}=$ $Q_{X}$, so that the first order action becomes

$$
I_{\mathrm{G}}\left[g ; b_{0}, H_{X}\right]=\int_{\gamma}\left(a-H_{X} d t\right)
$$

for some constant $X \in \mathfrak{g}$. The equations of motion are then equivalent to the vanishing of

$$
-i_{V} \Omega-d H_{X}=-\left(i_{V}+i_{v_{X}^{R}}\right) \Omega .
$$

By construction, this action is gauge invariant under the same assumptions as before. It is also invariant under the global transformations associated to $v_{X^{\prime}}^{R}$. Indeed, by using (2.12), one finds that this is the case for instance when $X^{\prime}(t)$ depends explicitly on time with an evolution determined by

$$
\frac{d X^{\prime}}{d t}=\operatorname{ad}_{X} X^{\prime}
$$

(ii) Another possibility uses an invariant symmetric tensor on $\mathfrak{g}^{*}$. Let $e_{a}$ denote the elements of a basis of $\mathfrak{g}$ and $e^{a}$ the elements of the dual basis. The Noether charges assocated to $e_{a}$ are $Q_{a}=-\left\langle b, e_{a}\right\rangle$. If $k^{a_{1} \ldots a_{m}}$ denote the components of the tensor, the Hamiltonian can be chosen to be,

$$
H_{k}=\frac{1}{m !} k^{a_{1} \ldots a_{m}} Q_{a_{1}} \ldots Q_{a_{m}}
$$

From (2.10) and (2.12), it follows that Lie derivatives with respect to $v_{\epsilon}^{L}$ and $v_{X^{\prime}}^{R}$ annihilate $H_{k}$. Thus, geometric actions supplemented by $H_{k}$ also preserve gauge and global symmetries, in this case with time independent $X^{\prime}$. An Hamiltonian quadratic in the Noether charges may be constructed for instance for semi-simple Lie algebras by using the inverse of the Killing form.

(iii) Another deformation of $I_{\mathrm{G}}\left[g, b_{0}\right]$ in (2.7) that changes the kinetic term is given by

$$
I_{\mathrm{G}}\left[g ; b_{0}, c\right]=I_{\mathrm{G}}\left[g, b_{0}\right]+\int_{\Sigma} \Omega_{\omega}, \quad \Omega_{\omega}=-\frac{1}{2} c \omega\left(\operatorname{Ad}_{g} \theta, \operatorname{Ad}_{g} \theta\right)
$$


with $\omega$ a Lie algebra 2-cocyle. Such a deformation is trivial in the sense that it can be absorbed by a redefinition of $b_{0}$ if $\omega$ is a coboundary. Non-trivial deformations are thus characterized by $[\omega] \in H^{2}(\mathfrak{g}, \mathbb{R})$.

These deformations modify the gauge symmetries: the requirement $i_{v_{\epsilon}^{L}} \widehat{\Omega}=0$ where $\widehat{\Omega}=\Omega+\Omega_{\omega}$ now restricts $\epsilon$ to solve

$$
\mathrm{ad}^{*}{ }_{\epsilon} b_{0}-\operatorname{cs}(\epsilon)=0
$$

with

$$
\langle s(X), Y\rangle=-\omega(X, Y) .
$$

Concerning global symmetries, the cocycle condition for $\omega$ implies that $d i_{v_{X}^{R}} \Omega_{\omega}=$ 0 . When taking into account that $i_{v_{X}^{R}} \Omega_{\omega}=-c \omega\left(\operatorname{Ad}_{g} X, \operatorname{Ad}_{g} \theta\right)=c\left\langle\operatorname{Ad}_{g^{-1}}^{*} s\left(\operatorname{Ad}_{\mathrm{g}} \theta\right), X\right\rangle$, it follows that, locally, there exists $S(g)$ such that $i_{v_{X}^{R}} \Omega_{\omega}=c d(\langle S(g), X\rangle)$. Hence, global symmetries are preserved by this deformation provided $S(g)$ exists globally. This is the case for instance when $H^{1}(G)=0$ or, as we will see in the next section, when $\omega$ originates from a group 2-cocycle in $G$. The associated Noether charges are

$$
\widehat{Q}_{X}=-\langle b-c S(g), X\rangle .
$$

They form a centrally extended representation of the symmetry algebra,

$$
£_{v_{X_{1}}^{R}} \widehat{Q}_{X_{2}}=\widehat{Q}_{\left[X_{1}, X_{2}\right]}+c \omega\left(X_{1}, X_{2}\right),
$$

and are gauge invariant under the modified gauge transformations, $£_{v_{\epsilon}^{L}} \widehat{Q}_{X}=0$.

When including a Hamiltonian $\widehat{H}_{X}=\widehat{Q}_{X}$, one now finds that all global symmetries generated by $v_{X^{\prime}}^{R}$ with time evolution determined by (2.15) survive if in addition $c \omega\left(X, X^{\prime}\right)=0$. When $c \neq 0$, this is a strong condition on allowed Hamiltonians respecting $G$-invariance.

For a Hamiltonian of the form $\widehat{H}_{k}=\frac{1}{m !} k^{a_{1} \ldots a_{m}} \widehat{Q}_{a_{1}} \ldots \widehat{Q}_{a_{m}}$, one has

$$
£_{v_{X^{\prime}}} H_{k}=\frac{1}{(m-1) !} c \omega_{b s} X^{\prime b} \widehat{Q}_{a_{2}} \cdots \widehat{Q}_{a_{m}} k^{s a_{2} \cdots a_{m}}
$$

where $\omega(X, Y)=\omega_{a b} X^{a} Y^{b}$. If we restrict ourselves to field independent Lie algebra elements $X^{\prime}$, invariance will hold in the quadratic case, $m=2$, for instance when

$$
\frac{d X^{\prime a}}{d t}=c X^{\prime b} \omega_{b s} k^{s a} .
$$

These deformations will be systematically discussed in the next section from the viewpoint of centrally extended groups. 


\section{Geometric actions for centrally extended groups}

The procedure outlined at the beginning of section 2 can be straightforwardly generalized for infinite dimensional groups and central extensions thereof. In applications to three-dimensional gravity, the asymptotic symmetry algebras of the theory is usually infinite dimensional, with central extensions in the representation through surface charges [1,44 46]. From the boundary point of view, one should thus study geometric actions associated to centrally extended groups.

\subsection{Central extensions}

A central extension of a group $G$ is a direct product $\widehat{G}=G \times \mathbb{R}$, whose elements are pairs $(g, m)$ with group operation $\left(g_{1}, m_{1}\right)\left(g_{2}, m_{2}\right)=\left(g_{1} g_{2}, m_{1}+m_{2}+\Xi\left(g_{1}, g_{2}\right)\right)$, where $\Xi: G \times G \rightarrow \mathbb{R}$ is a 2 -cocycle on $G$ that satisfies

$$
\Xi\left(g_{1} g_{2}, g_{3}\right)+\Xi\left(g_{1}, g_{2}\right)=\Xi\left(g_{1}, g_{2} g_{3}\right)+\Xi\left(g_{2}, g_{3}\right)
$$

which we assume for simplicitly to satisfy $\Xi(e, g)=0=\Xi(g, e)$. Two such central extensions denoted by $\Xi$ and $\Xi^{\prime}$ are isomorphic iff

$$
\Xi^{\prime}\left(g_{1}, g_{2}\right)=\Xi\left(g_{1}, g_{2}\right)+\mu\left(g_{1}\right)+\mu\left(g_{2}\right)-\mu\left(g_{1} g_{2}\right),
$$

where $\mu: G \rightarrow \mathbb{R}$. Denoting the elements of the corresponding centrally extended Lie algebra $\widehat{\mathfrak{g}}=\mathfrak{g} \oplus \mathbb{R}$ by $(X, n)$, the adjoint representation of $\widehat{G}$ can be written as

$$
\operatorname{Ad}_{(g, m)}(X, n)=\left(\operatorname{Ad}_{g} X, n-\langle S(g), X\rangle\right)
$$

where $S: G \rightarrow \mathfrak{g}^{*}$ is the Souriau cocycle on $G$ defined by

$$
\langle S(g), X\rangle=-\left.\frac{d}{d s}\left[\Xi\left(g, h(s) g^{-1}\right)+\Xi\left(h(s), g^{-1}\right)\right]\right|_{s=0},
$$

with differential at the identity given by

$$
\left.\frac{d}{d s} S(h(s))\right|_{s=0}=s(X) .
$$

Due to (3.1), it satisfies the 1-cocycle condition

$$
S\left(g_{1} g_{2}\right)=\operatorname{Ad}_{g_{2}-1}^{*} S\left(g_{1}\right)+S\left(g_{2}\right) .
$$

The adjoint action in $\hat{\mathfrak{g}}$ is given by

$$
\operatorname{ad}_{(X, n)}(Y, k)=\left(\operatorname{ad}_{X} Y, \omega(X, Y)\right),
$$


where (2.19) has been taken into account and where $s$ and $\operatorname{ad}_{X}$ are the differentials of $S$ and $\operatorname{Ad}_{g}$ at the identity respectively. Note that $s$ is entirely determined by the Lie algebra cocycle $[\omega] \in H^{2}(\mathfrak{g}, \mathbb{R})$ associated to $\Xi$ according to equation (2.19) .

Elements in $\widehat{\mathfrak{g}}^{*}$ are denoted by pairs $(b, c)$ where the dual element $c$ to the central extension of $\mathfrak{g}$ is the central charge. The pairing between $\widehat{\mathfrak{g}}$ and its dual space $\widehat{\mathfrak{g}}^{*}$ is defined by

$$
\langle(b, c),(X, n)\rangle=\langle b, X\rangle+c n,
$$

the coadjoint action is given by

$$
\operatorname{Ad}_{(g, m)}^{*}(b, c)=\left(\operatorname{Ad}_{g}^{*} b-c S\left(g^{-1}\right), c\right),
$$

while its the associated action in $\mathfrak{g}^{*}$ reads

$$
\operatorname{ad}_{(X, n)}^{*}(b, c)=\left(\operatorname{ad}_{X}^{*} b+c s(X), 0\right) .
$$

The extended Maurer-Cartan one-form is denoted by $\left(\theta, \theta_{\Xi}\right)$. The additonal piece is

$$
\theta_{\Xi}=d m+\left.\left[\delta_{2} \Xi\left(g_{1}, g_{2}\right)\right]\right|_{g_{1}=g^{-1}, g_{2}=g, \delta g_{2}=d g},
$$

where $\delta_{2}$ denotes an infinitesimal variation of $g_{2}$. Equation (2.5) is supplemented by

$$
d \theta_{\Xi}=\frac{1}{2}\langle s(\theta), \theta\rangle .
$$

Differentiating (3.6) with $g_{1}=g$ and $g_{2}=h(s)$ gives at $s=0$,

$$
d S(g)=-\operatorname{ad}_{\theta}^{*} S(g)+s(\theta) .
$$

By using (3.6) applied to $g h(s) g^{-1}$ and differentiating at $s=0$, with $X$ as in (2.1), one also gets

$$
\operatorname{ad}_{Y}^{*} S(g)=-\operatorname{Ad}_{g^{-1}}^{*} s\left(\operatorname{Ad}_{g} Y\right)+s(Y)
$$

where $Y=\operatorname{Ad}_{g^{-1}} X$. Combining (3.13) with (3.14) yields

$$
d S(g)=\operatorname{Ad}_{g^{-1}}^{*} s\left(\operatorname{Ad}_{g} \theta\right)
$$

\section{Remarks:}

(i) Suppose in particular that $H^{1}(\mathfrak{g}, \mathbb{R})=0$. It can then be shown that the Souriau map (2.19) on the level of the Lie algebra, $s: H^{2}(\mathfrak{g}, \mathbb{R}) \rightarrow H^{1}\left(\mathfrak{g}, \mathfrak{g}^{*}\right)$, $[\omega] \mapsto[s]$ is an isomorphism. If furthermore $H^{2}(\mathfrak{g}, \mathbb{R})$ is of dimension 1 and the Lie group $G$ is connected, (3.14) determines $S(g)$ uniquely from $\omega$ (see e.g. 35] and original references therein), without the need for an explicit expression for $\Xi$.

(ii) In the case of a centrally extended group, one can parametrize the elements of a coadjoint orbit by $(b, c)=\operatorname{Ad}_{(g, m)^{-1}}^{*}\left(b_{0}, c\right)$. For later use, note that, if $c \neq 0$ and

$$
b_{0} / c=-S(\Upsilon),
$$


for some group element $\Upsilon$, it follows from (3.6) that the coadjoint orbit generated from $\left(b_{0}, c\right)$ can also be generated from $(0, c)$ provided one changes $S(g)$ to $S(\Upsilon g)$ in the coadjoint action,

$$
\operatorname{Ad}_{(g, m)^{-1}}^{*}\left(b_{0}, c\right)=(-c S(\Upsilon g), c)
$$

\subsection{Geometric actions for central extensions}

The pre-symplectic potential for centrally extended groups is $a=\left\langle(b, c),\left(\theta, \theta_{\Xi}\right)\right\rangle$ and the kinetic term of the geometric action associated to a coadjoint orbit $O_{\left(b_{0}, c\right)}$ can be written as

$$
I_{\widehat{\mathrm{G}}}\left[g, m ; b_{0}, c\right]=I_{\mathrm{G}}\left[g ; b_{0}\right]+c \int\left(-\langle S(g), \theta\rangle+\theta_{\Xi}\right) .
$$

Using the relations of the previous section, the pre-symplectic 2-form $\widehat{\Omega}$ for centrally extended groups can then be worked out to be $\widehat{\Omega}=\Omega+\Omega_{\omega}$.

As compared to the analysis at the end of the previous section, the Lie algebra associated to the centrally extended group has an additional dimension consisting of vectors of the form $(0, n)$. The associated left invariant vector fields $v_{(0, n)}^{R}$ are global symmetries that generate constant shifts of $m$. They are all trivial however since these vectors belong to the extended little algebra. This can also be seen from the fact that

$$
I_{\widehat{\mathrm{G}}}\left[g, m ; b_{0}, c\right]=I_{\widehat{\mathrm{G}}}\left[g, 0 ; b_{0}, c\right],
$$

since the dependence on $m$ is only through a total time derivative that can be omitted. In the following we will simplify the notation and use $I_{\widehat{\mathrm{G}}}\left[g ; b_{0}, c\right]$. The additional Noether charges are trivial constants. More generally, the Noether charges can be choosen as

$$
Q_{(X, n)}=-i_{v_{(X, n)}^{R}}\left\langle\operatorname{Ad}_{(g, m)^{-1}}^{*}\left(b_{0}, c\right),\left(\theta, \theta_{\Xi}\right)\right\rangle=\widehat{Q}_{X}-c n,
$$

and now form an ordinary representation of the centrally extended symmetry algebra,

$$
£_{v_{\left(X_{1}, n_{1}\right)}^{R}} Q_{\left(X_{2}, n_{2}\right)}=Q_{\left[\left(X_{1}, n_{1}\right),\left(X_{2}, n_{2}\right)\right]} .
$$

For orbits generated by $\left(b_{0}, c\right)$ with $c \neq 0$ and where (3.16) holds, it follows from (3.17) and the left invariance of the Maurer-Cartan form that $I_{\mathrm{G}}\left[g ; b_{0}\right]$ can be absorbed into the term proportional to the central charge $c$, using a new a group element $u=\Upsilon g$,

$$
I_{\widehat{\mathrm{G}}}\left[g ; b_{0}, c\right]=c \int\left(-\langle S(u), \theta\rangle+\theta_{\Xi}\right)=I_{\widehat{\mathrm{G}}}[u ; 0, c] .
$$


and analogously, the charges (2.20) can be written as

$$
\widehat{Q}_{X}=\langle c S(u), X\rangle .
$$

This allows one to absorb the term proportional to the orbit representative $b_{0}$ also in geometric actions deformed by a Hamiltonian and to study the geometric actions corresponding to various coadjoint orbits in a unified fashion.

\subsection{Examples}

As a preparation for the cases of direct interest below, we briefly revisit in this subsection the well-known geometric actions for semi-simple loop groups $G$ and for the Virasoro group, first derived in [11,39]. More details can be found for instance in [36, 47].

\subsubsection{Kac-Moody groups}

\section{Loop groups and their extension}

Consider a finite dimensional simple and simply connected group $G$. The KacMoody group $\widehat{\mathrm{LG}}$ is given by the central extension of the loop group LG of $G$, whose elements are given by the continuous maps from the unit circle to $G$

$$
g: S^{1} \rightarrow G, \quad \varphi \mapsto g(\varphi),
$$

with $g(\varphi+2 \pi)=g(\varphi)$. In the same way, the loop algebra $L \mathfrak{g}$ corresponds to the algebra of continuous maps from $S^{1}$ to $\mathfrak{g}$. The pairing between $\mathrm{L} \mathfrak{g}$ and its dual $\mathrm{L} \mathfrak{g}^{*}$ reads

$$
\langle b(\varphi), X(\varphi)\rangle=\int_{0}^{2 \pi} d \varphi \operatorname{Tr}[b(\varphi) X(\varphi)],
$$

where $\operatorname{Tr}$ denotes the normalized Killing form. The central extension is determined by the 2-cocycle on the loop group

$$
\Xi\left(g_{1}, g_{2}\right)=\frac{1}{4 \pi} \int_{\bar{D}} \operatorname{Tr}\left[g_{1}^{-1} \bar{d} g_{1} \bar{d} g_{2} g_{2}^{-1}\right]
$$

where $\bar{d}$ denotes the exterior derivative on the disk $\bar{D}$ whose boundary is $S^{1}$. The 1 - cocycle defining the adjoint action can then be obtained from (3.4),

$$
S(g)=\frac{1}{2 \pi} g^{-1} \partial_{\varphi} g
$$

with $s(X)=\frac{1}{2 \pi} \partial_{\varphi} X$. Equation (3.11) gives

$$
\theta_{\Xi}=d m(\varphi)+\frac{1}{4 \pi}\left(\int_{0}^{2 \pi} d \varphi \operatorname{Tr}\left[g^{-1} \partial_{\varphi} g g^{-1} d g\right]+\int_{\bar{D}} \operatorname{Tr}\left[g^{-1} \bar{d} g g^{-1} \bar{d} g g^{-1} d g\right]\right) .
$$




\section{Geometric actions}

The geometric action (3.18) therefore turns out to be

$$
I_{\widehat{\mathrm{LG}}}\left[g ; b_{0}, c\right]=\iint_{0}^{2 \pi} d \varphi \operatorname{Tr}\left[b_{0} d g g^{-1}-\frac{c}{4 \pi} g^{-1} \partial_{\varphi} g g^{-1} d g\right]+c \Gamma .
$$

where

$$
\Gamma=\frac{1}{4 \pi} \iint_{\bar{D}} \operatorname{Tr}\left[g^{-1} \bar{d} g g^{-1} \bar{d} g g^{-1} d g\right] .
$$

Using the notation $d=d t \partial_{t}$ and defining a manifold $M=\gamma \times \bar{D}$ where $t$ is the coordinate along $\gamma$, the Wess-Zumino term $\Gamma$ can be put into the standard form

$$
\Gamma=\frac{1}{12 \pi} \int_{M} \operatorname{Tr}\left[\left(d^{T} g g^{-1}\right)^{3}\right]
$$

where $d^{T}$ denotes the exterior derivative on the whole of $M, d t \wedge d \varphi \wedge d r$ is considered as orientation for the integration on $M$, with boundary conditions such that the only contribution from $\partial M$ arise from $\gamma \times S^{1}$.

According to (3.20), the Noether charges associated to the symmetries corresponding to right multiplication by group elements $(g(\varphi), m(\varphi))$ and generated by $(X(\varphi), n(\varphi))$ are

$$
Q_{(X, n)}=\int_{0}^{2 \pi} d \varphi(\operatorname{Tr}[Q(\varphi) X(\varphi)]-c n(\varphi)), \quad Q(\varphi)=\frac{c}{2 \pi} g^{-1} \partial_{\varphi} g-g^{-1} b_{0} g .
$$

In order to make contact with 3d gravity, we will choose the following bilinear combination of $Q$ as a Hamiltonian,

$$
H_{2}=\frac{\pi}{c} \int d \varphi \operatorname{Tr}\left[Q^{2}\right]
$$

Indeed, this Hamiltonian arises from the Chern-Simons formulation of $\mathrm{AdS}_{3}$ gravity when imposing Brown-Henneaux boundary conditions [2]. Under global symmetries generated by $v_{X^{\prime}}^{R}$, it transforms as

$$
£_{v_{X^{\prime}}^{R}} H_{2}=\int d \varphi \operatorname{Tr}\left[Q \partial_{\varphi} X^{\prime}\right]
$$

Since under the same transformation $\delta_{X^{\prime}} I_{\widehat{\mathrm{LG}}}=\int d t d \varphi \operatorname{Tr}\left[Q \partial_{t} X^{\prime}\right]$, it follows that

$$
I_{\widehat{\mathrm{LG}}}\left[g ; b_{0}, c, H_{2}\right]=I_{\widehat{\mathrm{LG}}}\left[g ; b_{0}, c\right]-\int d t H_{2}
$$

is invariant under the global symmetries generated by $X^{\prime}$ if $X^{\prime}(t, \varphi)=X^{\prime}(t+\varphi)$. 


\section{Relation to chiral WZW theories}

Defining now $2 \partial_{-}=\partial_{t}-\partial_{\varphi}$, we can write

$$
I_{\widehat{\mathrm{LG}}}\left[g ; b_{0}, c, H_{2}\right]=-\int d t d \varphi \operatorname{Tr}\left[2 b_{0} g^{-1} \partial_{-} g\right]+I_{\mathrm{WZW}}[g ; c],
$$

where $I_{\mathrm{WZW}}[g ; c]$ corresponds to the chiral WZW model

$$
I_{\mathrm{WZW}}[g ; c]=\frac{c}{2 \pi} \int d t d \varphi \operatorname{Tr}\left[g^{-1} \partial_{\varphi} g g^{-1} \partial_{-} g\right]+c \Gamma,
$$

after neglecting a time independent $\operatorname{Tr} b_{0}^{2}$ in the integrand. In the particular case of a $\varphi$ independent $b_{0}$, action (3.36) has been obtained in [8] after solving the constraints of Chern-Simons theory based on a semisimple group $G$ on a spatial disk with a source. In the context of $\mathrm{AdS}_{3}$ gravity it has been used in [48] and more recently in [49].

The term proportional to $b_{0}$ can be absorbed into the chiral WZW model by considering a group element $\Upsilon=\Upsilon(\varphi)$ that solves equation (3.16), which in this case takes the form

$$
\Upsilon^{-1} \partial_{\varphi} \Upsilon=-\frac{2 \pi}{c} b_{0}
$$

The action (3.29) can then be written as a chiral WZW action for a non periodic field $u=\Upsilon g$, i.e.,

$$
I_{\widehat{\mathrm{LG}}}\left[g ; b_{0}, c, H_{2}\right]=I_{\mathrm{WZW}}[u, c] .
$$

In this formulation, the dependence on the orbit representative $b_{0}$ is translated into a nontrivial periodicity of the field $u$,

$$
u(\varphi+2 \pi)=\mathcal{M}\left(b_{0}\right) u(\varphi), \quad \mathcal{M}\left(b_{0}\right)=\mathcal{P} \exp \left[-\frac{2 \pi}{c} \oint d \varphi b_{0}\right] .
$$

\subsubsection{Virasoro Group}

\section{Diffeomorphism group and its extension}

The Virasoro group is the central extension of Diff $\left(S^{1}\right)$, which in turn corresponds to the orientation-preserving diffeomorphism group of the circle with elements $f$ satisfying

$$
f(\varphi+2 \pi)=f(\varphi)+2 \pi, \quad f^{\prime}>0 .
$$

The associated Lie algebra will be denoted by $\operatorname{Vec}\left(S^{1}\right)$. Its elements are vector fields on the circle $X=X(\varphi) \partial_{\varphi}$, while elements of the dual $\operatorname{Vec}\left(S^{1}\right)^{*}$ are taken as quadratic differentials on $S^{1}, b=b(\varphi)(d \varphi)^{2}$. The natural pairing between $\operatorname{Vec}\left(S^{1}\right)$ and its dual is

$$
\langle b, X\rangle=\int_{0}^{2 \pi} d \varphi b(\varphi) X(\varphi) .
$$


The adjoint and coadjoint actions of Diff $\left(S^{1}\right)$ are

$$
\operatorname{Ad}_{f^{-1}} X=\frac{1}{f^{\prime}(\varphi)} X(f(\varphi)) \partial_{\varphi}, \quad \operatorname{Ad}_{f^{-1}}^{*} b=f^{\prime}(\varphi)^{2} b(f(\varphi))(d \varphi)^{2},
$$

The associated infinitesimal adjoint action is minus the Lie bracket for vector fields on $S^{1}$.

The 2-cocycle determining the Virasoro group is the Thurston-Bott cocycle

$$
\Xi\left(f_{1}, f_{2}\right)=-\frac{1}{48 \pi} \int_{0}^{2 \pi} d \varphi \log \left(\partial_{\varphi} f_{1} \circ f_{2}\right) \partial_{\varphi}\left(\log \left(\partial_{\varphi} f_{2}\right)\right) .
$$

One then finds the Schwarzian derivative (1.3) as the corresponding Souriau cocycle, with differential at the identity given by

$$
s(X)=\frac{1}{24 \pi} X^{\prime \prime \prime}(\varphi)
$$

while the Maurer-Cartan form is

$$
\left(\theta, \theta_{\Xi}\right)=\left(\frac{d f}{f^{\prime}} \partial_{\varphi}, d m+\frac{1}{48 \pi} \int_{0}^{2 \pi} d \varphi \frac{d f}{f^{\prime}}\left(\frac{f^{\prime \prime}}{f^{\prime}}\right)^{\prime}\right) .
$$

\section{Geometric actions}

The geometric action (3.18) for the Virasoro group is then found to be

$$
I_{\widehat{\operatorname{Diff}}\left(S^{1}\right)}\left[f ; b_{0}, c\right]=\int d \varphi d t\left[b_{0}(f) f^{\prime} \dot{f}+\frac{c}{48 \pi} \frac{\dot{f}^{\prime \prime}}{f^{\prime}}\right] .
$$

The next step is to add a Hamiltonian preserving diffeomorphisms on the circle. Again, in order to make contact with three-dimensional gravity, we chose an Hamiltonian associated with a suitable vector field. From the discussion of section 2 after equation (2.21), it follows that we may choose $X=-\partial_{\varphi}$ with associated Noether charge

$$
H_{1}=\int d \varphi\left[b_{0}(f) f^{\prime 2}+\frac{c}{48 \pi} \frac{f^{\prime \prime 2}}{f^{\prime 2}}\right]
$$

The invariance under diffeomorphisms $\delta_{X^{\prime}} f=X^{\prime} \partial_{\varphi} f$ of (3.47) then survives the deformation $H_{1} d t$ if $X^{\prime}$ evolves according to (2.15). This becomes explicitly $\partial_{t} X^{\prime}=$ $\operatorname{ad}_{-\partial_{\varphi}} X^{\prime}=\partial_{\varphi} X^{\prime}$, and implies $X^{\prime}=X^{\prime}(t+\varphi)$. At this point, it is convenient to define $\partial_{\varphi} f=e^{\phi}$ with $\phi(\varphi+2 \pi)=\phi(\varphi)$. This can be done because of (3.41). In that case, after adding the Hamiltonian $H_{1}$ to (3.47), we find

$$
I_{\widehat{\operatorname{Diff}}\left(S^{1}\right)}\left[f ; b_{0}, c, H_{1}\right]=2 \int d \varphi d t\left[b_{0}(f) f^{\prime} \partial_{-} f+\frac{c}{48 \pi} \phi^{\prime} \partial_{-} \phi\right] .
$$




\section{Relation to chiral bosons}

One may again eliminate the term proportional to the representative $b_{0}(\varphi)$ from the action by defining a new field with a suitable periodicity. Following section 3 , this can be done by defining a new field $F=\Upsilon \circ f$ where $\Upsilon$ satisfies

$$
c S_{\varphi}[\Upsilon]=-b_{0}(\varphi)
$$

The ansatz $F=e^{\mu(f)}$ turns this equation into

$$
\frac{c}{48 \pi}\left(\frac{d \mu}{d f}\right)^{2}-c S_{f}[\mu]=b_{0}(f) .
$$

In terms of the new field $F$, the action (3.49) reduces to $I_{\widehat{\operatorname{Diff}}\left(S^{1}\right)}[(F, 0) ;(0, c)]$, which can be rewritten as the action of a chiral boson $\chi$

$$
I_{\mathrm{cB}}[\chi ; c]=\frac{c}{24 \pi} \int d t d \varphi \partial_{\varphi} \chi \partial_{-} \chi
$$

where $\partial_{\varphi} F=e^{\chi}$. In this case, the field redefinition that relates (3.52) with (3.49) is

$$
\chi=\mu(f)+\phi+\log \left(\frac{d \mu}{d f}\right) .
$$

As a side remark, note that (3.51) turns out to be Hill's equation: defining $\psi(f)=$ $\left(\partial_{\varphi} f\right)^{1 / 2} \exp (-\chi / 2),(3.51)$ becomes

$$
\left(-\frac{c}{12 \pi} \partial_{f}^{2}+b_{0}(f)\right) \psi(f)=0 .
$$

It is well-known that conjugacy classes of monodromy matrices associated to the Hill's equation characterize Virasoro coadjoint orbits (see e.g. [50]).

\section{Geometric action for semi-direct products}

\subsection{Semi-direct product groups}

A semidirect product of a semi-simple Lie group $G$ and an abelian group $\mathcal{A}$, under some representation $\sigma$ of $G$ on $\mathcal{A}$,

$$
\mathcal{S}_{\sigma}=G \ltimes_{\sigma} \mathcal{A}
$$

is a group with elements of the form $(g, \alpha)$, where $g \in G$ and $\alpha \in A$. The group operation is given by $\left(g_{1}, \alpha_{1}\right)\left(g_{2}, \alpha_{2}\right)=\left(g_{1} g_{2}, \alpha_{1}+\sigma_{g_{1}} \alpha_{2}\right)$. As $\mathcal{A}$ is abelian, its Lie algebra is isomorphic to itself and therefore the Lie algebra associated to $\mathcal{S}_{\sigma}$ is given 
by $\mathfrak{s}=\mathfrak{g} \oplus \mathcal{A}$. Denoting the elements of $\mathfrak{s}$ by $(X, \alpha)$, and the elements of its dual space by $(j, p)$, the bilinear form on $\mathfrak{s}$ is

$$
\langle(j, p),(X, \alpha)\rangle=\langle j, X\rangle+\langle p, \alpha\rangle_{\mathcal{A}}
$$

where $\langle$,$\rangle and \langle,\rangle_{\mathcal{A}}$ are the natural pairings in $\mathfrak{g}$ and $\mathcal{A}$ respectively. The adjoint and coadjoint actions of $\mathcal{S}$ on $\mathfrak{s}$ and $\mathfrak{s}^{*}$ [51] follow from (2.1) and (2.2),

$$
\begin{aligned}
\operatorname{Ad}_{(g, \alpha)}(X, \beta) & =\left(\operatorname{Ad}_{g} X, \sigma_{g} \beta-\Sigma_{\operatorname{Ad}_{g} X} \alpha\right), \\
\operatorname{Ad}_{(g, \alpha)}^{*}(j, p) & =\left(\operatorname{Ad}_{h}^{*} j+\sigma_{g}^{*} p \odot \alpha, \sigma_{g}^{*} p\right),
\end{aligned}
$$

where $\Sigma$ is the infinitesimal form of $\sigma, p \odot \alpha$ is defined as

$$
\langle p \odot \alpha, X\rangle=\left\langle p, \Sigma_{X} \alpha\right\rangle_{\mathcal{A}}=-\left\langle\Sigma_{X}^{*} p, \alpha\right\rangle_{\mathcal{A}}
$$

and $\sigma^{*}, \Sigma^{*}$ are the dual maps of $\sigma, \Sigma$ respectively (with respect to the pairing $\langle,\rangle_{\mathcal{A}}$ ). The commutation relations for $\mathfrak{s}$ are defined by the infinitesimal form of (4.3), i.e.,

$$
[(X, \alpha),(Y, \beta)]=\operatorname{ad}_{(X, \alpha)}(Y, \beta)=\left(\operatorname{ad}_{X} Y, \Sigma_{X} \beta-\Sigma_{Y} \alpha\right)
$$

We will construct geometric actions for the group $\mathcal{S}_{\sigma}$ when $\sigma$ corresponds to the adjoint representation and $\mathcal{A}$ is given by the Lie algebra of $G$ seen as an abelian vector space, which will be denoted by $\mathfrak{g}_{a b}$. Using (4.3), the adjoint action of $\mathcal{S}_{\text {Ad }}=$ $G \ltimes_{\text {Ad }} \mathfrak{g}_{a b}$ takes the form

$$
\operatorname{Ad}_{(g, \alpha)}(Y, \beta)=\left(\operatorname{Ad}_{g} Y, \operatorname{Ad}_{g} \beta-\operatorname{ad}_{\mathrm{Ad}_{g} Y} \alpha\right),
$$

while its infinitesimal form becomes

$$
\operatorname{ad}_{(X, \alpha)}(Y, \beta)=\left(\operatorname{ad}_{X} Y, \operatorname{ad}_{X} \beta-\operatorname{ad}_{Y} \alpha\right)
$$

For the coadjoint action, (4.4) leads to [23,24]

$$
\operatorname{Ad}_{(g, \alpha)^{-1}}^{*}(j, p)=\left(\operatorname{Ad}_{g^{-1}}^{*} j-\operatorname{Ad}_{g^{-1}}^{*} \operatorname{ad}_{\alpha}^{*} p, \operatorname{Ad}_{g^{-1}}^{*} p\right)
$$

The Maurer-Cartan one-form consists of a pair $\left(\theta, \theta_{\alpha}\right)$, where $\theta$ is defined in (2.5) and

$$
\theta_{\alpha}=\operatorname{Ad}_{g^{-1}} d \alpha
$$

\subsection{Geometric actions for $\mathcal{S}_{\mathrm{Ad}}$}

The geometric action (2.7) for a semi-direct product with an adjoint action is given by

$$
I_{\mathcal{S}_{\mathrm{Ad}}}\left[g, \alpha ; p_{0}, j_{0}\right]=I_{\mathrm{G}}\left[g ; j_{0}\right]-I_{\mathrm{G}}\left[g ; \operatorname{ad}_{\alpha}^{*} p_{0}\right]
$$


In terms of $\left(h_{L}, \alpha_{L}\right)$ and $\left(h_{R}, \alpha_{R}\right)$, left and right actions in a semi-direct product group act as

$$
\begin{aligned}
& g \rightarrow h_{L} g, \quad \alpha \rightarrow \alpha_{L}+\operatorname{Ad}_{h_{L}} \alpha, \\
& g \rightarrow g h_{R}, \quad \alpha \rightarrow \alpha+\operatorname{Ad}_{g} \alpha_{R} .
\end{aligned}
$$

Geometric actions (4.10) are invariant under gauge and global transformations generated by vector fields $v_{(X, n)}^{R}$ and $v_{(\epsilon, \zeta)}^{L}$ respectively. They are given by

$$
\begin{aligned}
v_{(X, v)}^{R} & =\left.\frac{d}{d s}\left(g h_{R}(s), \alpha+\operatorname{Ad}_{g} \alpha_{R}(s)\right)\right|_{s=0}, X=\left.\frac{d}{d s} h_{R}(s)\right|_{s=0}, v=\left.\frac{d}{d s} \alpha_{R}(s)\right|_{s=0}, \\
v_{(\epsilon, \zeta)}^{L} & =\left.\frac{d}{d s}\left(h_{L}(s) g, \alpha_{L}(s)+\operatorname{Ad}_{h_{L}(s)} \alpha\right)\right|_{s=0}, \epsilon=\left.\frac{d}{d s} h_{L}(s)\right|_{s=0}, \zeta=\left.\frac{d}{d s} \alpha_{L}(s)\right|_{s=0} .
\end{aligned}
$$

where $(\epsilon, \zeta)$ depends arbitrary on time $t$ and belongs to the little algebra of the representatives $\left(p_{0}, j_{0}\right)$.

\subsection{Geometric action for centrally extended $\widehat{\mathcal{S}}_{\mathrm{Ad}}$}

Let us consider now the centrally extended group $\widehat{\mathcal{S}}=\widehat{G} \ltimes_{\text {Ad }} \widehat{\mathfrak{g}}_{a b}$, i.e. a central extension of a semi-direct product group under the adjoint action, whose elements will be denoted by $\left(g, m_{1}, \alpha, m_{2}\right)$. The elements of the algebra $\widehat{\mathfrak{s}}$ will be denoted by $\left(X, n_{1}, \alpha, n_{2}\right)$, while the elements of the dual by $\left(j, c_{1}, p, c_{2}\right)$. The coadjoint action as well as for the geometric action are constructed using (4.8) and (4.10) and replacing

$$
j_{0} \rightarrow\left(j_{0}, c_{1}\right), \quad p_{0} \rightarrow\left(p_{0}, c_{2}\right), \quad \operatorname{Ad}_{g}^{*} \rightarrow \operatorname{Ad}_{\left(g, m_{1}\right)}^{*}, \quad \operatorname{ad}_{\alpha}^{*} \rightarrow \operatorname{ad}_{\left(\alpha, n_{2}\right)}^{*},
$$

where $\operatorname{Ad}_{\left(g, m_{1}\right)}^{*}$ is defined in (3.9) and $\operatorname{ad}_{\left(\alpha, n_{2}\right)}^{*}$ corresponds to its infinitesimal form given by (3.10). In the same way, the one-form $\left(\theta, \theta_{\alpha}\right)$ introduced in the previous section must be replaced by $\left(\theta, \theta_{\Xi}, \theta_{\alpha}, \theta_{\omega}\right)$ where $\theta_{\omega}=\left\langle S(g), \theta_{\alpha}\right\rangle$.

The geometric action on a coadjoint orbit $O_{\left(j_{0}, c_{1}, p_{0}, c_{2}\right)}$ is given by the centrally extended version of (4.10),

$$
I_{\widehat{\mathcal{S}}_{\mathrm{Ad}}}\left[g, \alpha ; p_{0}, j_{0}, c_{1}, c_{2}\right]=I_{\widehat{\mathrm{G}}}\left[g ; j_{0}, c_{1}\right]-I_{\mathrm{G}}\left[g ; \operatorname{ad}_{\alpha}^{*} p_{0}+c_{2} s(\alpha)\right],
$$

where $I_{\widehat{\mathrm{G}}}$ is given by (3.18).

As before, the orbit representatives $j_{0}$ and $p_{0}$ can be absorbed into the terms proportional to the central charges by defining suitable fields $u=\Upsilon g$ and $a=$ $\eta+A_{\Upsilon} \alpha$. Using (3.14) allows one to write the action (4.13) in the form

$$
\begin{aligned}
& I_{\widehat{\mathcal{S}}_{\mathrm{Ad}}}\left[g, \alpha ; p_{0}, j_{0}, c_{1}, c_{2}\right]=I_{\mathcal{S}}\left[g, \alpha ; j_{0}, p_{0}\right]+c_{1} \int\left\langle\operatorname{Ad}_{g^{-1}}^{*} S(\Upsilon), \theta\right\rangle \\
& \quad+c_{2} \int\left\langle\operatorname{Ad}_{g^{-1}}^{*}\left(\operatorname{Ad}_{\Upsilon^{-1}}^{*} s(\eta)-\operatorname{ad}_{\alpha}^{*} S(\Upsilon)\right), \theta\right\rangle+I_{\widehat{\mathcal{S}}_{\mathrm{Ad}}}\left[u, a ; 0,0, c_{1}, c_{2}\right] .
\end{aligned}
$$


An inspection of the latter expression makes evident that, provided the pair $(\Upsilon, \eta)$ satisfies

$$
c_{2} S(\Upsilon)=-p_{0}, \quad c_{2} \operatorname{Ad}_{\Upsilon-1}^{*} s(\eta)=-j_{0}+\frac{c_{1}}{c_{2}} p_{0},
$$

the geometric action reduces to

$$
I_{\widehat{\mathcal{S}}_{\mathrm{Ad}}}\left[g, \alpha ; p_{0}, j_{0}, c_{1}, c_{2}\right]=I_{\widehat{\mathcal{S}}_{\mathrm{Ad}}}\left[u, a ; 0,0, c_{1}, c_{2}\right] .
$$

As we will see in the next examples, the latter equality will allow us to link geometric actions based on groups having a semi-direct product structure with actions appearing in the Hamiltonian reduction of $3 \mathrm{~d}$ gravity in the case of vanishing cosmological constant.

\subsection{Examples}

\subsubsection{Loop groop of $G \ltimes \mathfrak{g}$ and its extension}

Let us consider the group $\widehat{\mathcal{S}}_{\mathrm{Ad}}=\widehat{L G} \ltimes_{\mathrm{Ad}} \widehat{\mathrm{Lg}}_{\mathrm{ab}}$, where $G$ is a semi-simple Lie group. Its elements are of the form $\left(g(\varphi), m_{1}, \alpha(\varphi), m_{2}\right)$, where $g(\varphi)$ is a map of the form (3.24), $\alpha$ is an element the loop algebra $\mathrm{Lg}$ and $m_{1}, m_{2}$ correspond to the central extensions of $\mathrm{L} G$ and $\mathrm{L} \mathfrak{g}$ respectively. The geometric action can be obtained directly from (4.13) using the machinery developed in subsection 3.3.1,

$$
\begin{aligned}
& I_{\widehat{L G} \ltimes \widehat{A d}_{\mathrm{Lg}_{\mathrm{ab}}}}\left[g, \alpha ; p_{0}, j_{0}, c_{1}, c_{2}\right]= \\
& I_{\widehat{\mathrm{LG}}}\left[g ; j_{0}, c_{1}\right]-\int d \varphi \operatorname{Tr}\left[\left(\left[\alpha, p_{0}\right]+\frac{c_{2}}{2 \pi} \alpha^{\prime}\right) d g g^{-1}\right],
\end{aligned}
$$

where $I_{\widehat{\mathrm{LG}}}$ is given by (3.29). From (4.11), this action is invariant under right multiplication of $g$, but also under the adjoint action on $\alpha$, i.e., under

$$
\delta_{(X, v)}(g, \alpha)=\left(g X(\varphi), g v(\varphi) g^{-1}\right)
$$

These global symmetries give rise to the following Noether charges

$$
\begin{aligned}
& \mathcal{J}_{X}=\int_{0}^{2 \pi} d \varphi \operatorname{Tr}[X j], \quad j=\frac{c_{1}}{2 \pi} g^{-1} \partial_{\varphi} g-g^{-1}\left(j_{0}-\frac{c_{2}}{2 \pi} \partial_{\varphi} \alpha-\left[\alpha, p_{0}\right]\right) g, \\
& \mathcal{P}_{v}=\int_{0}^{2 \pi} d \varphi \operatorname{Tr}[v p], \quad p=\frac{c_{2}}{2 \pi} g^{-1} \partial_{\varphi} g-g^{-1} p_{0} g .
\end{aligned}
$$

A Hamiltonian motivated by asymptotically flat gravity in three dimensions is

$$
H_{2}=\frac{\pi}{c_{2}} \int d \varphi \operatorname{Tr}\left[p^{2}\right]
$$


Since $H_{2}$ transforms as (3.34) under the symmetries (4.18) and $\delta_{(X, v)} I_{\widehat{L G} \ltimes_{\mathrm{Ad}} \widehat{\mathrm{Lg}}_{\mathrm{ab}}}$ is given by $\int d t d \varphi \operatorname{Tr}\left[\partial_{t} v p+\partial_{t} X j\right]$, one concludes that in presence of the Hamiltonian, action

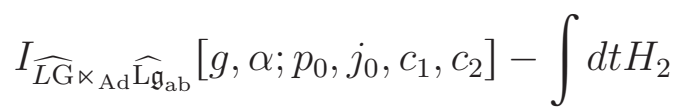

is invariant provided $X=X_{0}(\varphi), v=v_{0}(\varphi)+t \partial_{\varphi} X_{0}$.

\section{Relation to Flat WZW model}

The terms in (4.17) proportional to the orbit representatives $j_{0}$ and $p_{0}$ can be absorbed into the kinetic term of the flat WZW model by defining new fields $u=\Upsilon g$ and $a=\eta+\Upsilon \alpha \Upsilon^{-1}$ satisfying equations (4.15), which in this case take the form

$$
-\frac{c_{2}}{2 \pi} \Upsilon^{-1} \partial_{\varphi} \Upsilon=p_{0}, \quad-\frac{c_{2}}{2 \pi} \Upsilon^{-1} \partial_{\varphi} \eta \Upsilon=j_{0}-\frac{c_{1}}{c_{2}} p_{0}
$$

After including the Hamiltonian, the geometric action (4.21) can be written in terms of the new fields $u$ and $a$ as

$$
\begin{aligned}
& I_{\widehat{L G} \ltimes_{\mathrm{Ad}} \widehat{\mathrm{Lg}}_{\mathrm{ab}}}\left[g, \alpha ; p_{0}, j_{0}, c_{1}, c_{2}\right]= \\
& I_{\widehat{\mathrm{LG}}}\left[u ; 0, c_{1}\right]-\frac{c_{2}}{2 \pi} \int d \varphi d t \operatorname{Tr}\left[\dot{u} u^{-1} a^{\prime}-\frac{1}{2}\left(u^{-1} u^{\prime}\right)^{2}\right] .
\end{aligned}
$$

This corresponds to the flat WZW model obtained in [38, 52] in the context of asymptotically flat three-dimensional Einstein gravity. In this representation, the information on $\left(p_{0}, j_{0}\right)$ is encoded in the periodicity of the fields $u$ and $a$,

$$
u(\varphi+2 \pi)=\mathcal{M}\left(p_{0}\right) u(\varphi), \quad a(\varphi+2 \pi)=\mathcal{M}\left(p_{0}\right) a(\varphi) \mathcal{M}^{-1}\left(p_{0}\right)+\mathcal{N}\left(j_{0}, p_{0}\right),
$$

where $\mathcal{M}\left(p_{0}\right)$ is given by (3.40) and $\mathcal{N}\left(j_{0}, p_{0}\right)=-\frac{2 \pi}{c_{2}} \oint \Upsilon\left(j_{0}-\frac{c_{1}}{c_{2}} p_{0}\right) \Upsilon^{-1}$.

\subsection{2 $\widehat{\mathrm{BMS}}_{3}$ group}

The $\widehat{\mathrm{BMS}}_{3}$ group is the semidirect product of the Virasoro group and its algebra (seen as an abelian vector space) under the adjoint action

$$
\widehat{\mathrm{BMS}}_{3}=\widehat{\operatorname{Diff}}\left(S^{1}\right) \ltimes \operatorname{Vec}\left(S^{1}\right)_{\mathrm{ab}} .
$$

Its elements are pairs $(f, \alpha)$, where $f$ is a diffeomorphism of the circle (3.41) and $\alpha$ satisfies

$$
\alpha(\varphi+2 \pi)=\alpha(\varphi)
$$


Therefore, the corresponding geometric action has the form (4.13) where the coadjoint action is the one of the Virasoro group (3.43) and $S$ is the Schwarzian derivative (1.3). The resulting action is

$$
\begin{aligned}
& I_{\widehat{\mathrm{BMS}_{3}}}\left[f, \alpha ; p_{0}, j_{0}, c_{1}, c_{2}\right]= \\
& \quad I_{\widehat{\operatorname{Diff}}\left(S^{1}\right)}\left[f ; j_{0}, c_{1}\right]+\int d \varphi\left[f^{\prime} d f\left(p_{0}^{\prime} \alpha+2 p_{0} \alpha^{\prime}-\frac{c_{2}}{24 \pi} \alpha^{\prime \prime \prime}\right) \circ f\right] .
\end{aligned}
$$

Defining $e^{\phi}=f^{\prime}, \xi=\alpha^{\prime}(f)$, the geometric action on a orbit of the $\widehat{\mathrm{BMS}}_{3}$ group takes the form

$$
\begin{aligned}
& I_{\widehat{\mathrm{BMS}}_{3}}\left[f, \alpha ; p_{0}, j_{0}, c_{1}, c_{2}\right]= \\
& I_{\widehat{\operatorname{Diff}}\left(S^{1}\right)}\left[f ; j_{0}, c_{1}\right]+\int d \varphi d t\left[f^{\prime} \dot{f}\left(p_{0}^{\prime} \alpha+2 p_{0} \alpha^{\prime}\right) \circ f+\frac{c_{2}}{24 \pi} \dot{\phi} \xi^{\prime}\right] .
\end{aligned}
$$

From (4.11), we can infer that the global transformations laws of the fields are

$$
\delta_{(X, v)}(f, \alpha(f))=\left(X(\varphi) \partial_{\varphi} f, v(\varphi) \partial_{\varphi} f\right)
$$

We will choose the Hamiltonian as the charge associated to rigid translation, $(X, v)=$ $\left(0,-\partial_{\varphi}\right)$

$$
H=\int_{0}^{2 \pi} d \varphi\left[f^{\prime 2} p_{0}(f)+\frac{c_{2}}{48 \pi} \frac{f^{\prime \prime 2}}{f^{\prime 2}}\right] .
$$

As in the previous examples, this choice is inspired by three-dimensional Einstein gravity without cosmological constant. Let us now consider the geometric action (4.27) deformed by the Hamiltonian (4.29). Symmetry (4.28) will be preserved in this new action provided the suitable extension of (2.15) is satisfied, i.e. $\partial_{t}\left(X^{\prime}, v^{\prime}\right)=$ $\operatorname{ad}_{\left(0,-\partial_{\varphi}\right)}\left(X^{\prime}, v^{\prime}\right)$, which gives $X^{\prime}=X_{0}(\varphi)$ and $v^{\prime}=v_{0}(\varphi)+t \partial_{\varphi} X_{0}$.

\section{Relation to chiral $\mathrm{BMS}_{3}$ theory}

Defining new fields $F=\Upsilon \circ f$ and $a=\eta+\operatorname{Ad}_{\Upsilon} \alpha$ satisfying (4.15), which in this case takes the form

$$
c_{2} S_{\varphi}[\Upsilon]=-p_{0}, \quad-\frac{c_{2}}{24 \pi} \Upsilon^{\prime 2} \eta^{\prime \prime \prime}(\Upsilon)=j_{0}-\frac{c_{1}}{c_{2}} p_{0},
$$

the terms in (4.27) proportional to the orbit representatives $j_{0}$ and $p_{0}$ can be absorbed in this field redefinition. Including $H$ in (4.27), the geometric action for the $\mathrm{BMS}_{3}$ group can be written as

$$
I_{\widehat{\mathrm{BMS}}}\left[f, \alpha ; p_{0}, j_{0}, c_{1}, c_{2}, H\right]=I_{\widehat{\operatorname{Diff}}\left(S^{1}\right)}\left[\chi ; 0, c_{1}\right]+\frac{c_{2}}{24 \pi} \int d \varphi d t\left(\dot{\chi} \zeta^{\prime}-\frac{1}{2} \chi^{\prime 2}\right)
$$


where $I_{\widehat{\operatorname{Diff}}\left(S^{1}\right)}[\chi ; 0]$ is given in (3.52),$\chi=\log \left(\partial_{\varphi} F\right)$ and $\zeta=a^{\prime}(F)$. This is the chiral $\mathrm{BMS}_{3}$ model constructed as the classical dual of three-dimensional asymptotically flat gravity [38, 52] (See also [53] for a higher spin extension).

Note that, as in the Virasoro case, the first relation of (4.30) produces the Hill's equation for the variable $\psi(f)$ defined in (3.54) with $\left(p_{0}, c_{2}\right)$ playing the role of $\left(b_{0}, c\right)$. In the same way, the second equation of (4.30) controls the orbits associated to the pair $\left(j_{0}, c_{1}\right)$.

\section{$5 \quad$ Discussion and perspectives}

We have studied geometric actions for various groups arising in three-dimensional gravity. In a first stage, we have analyzed geometric actions for loop groups reobtaining (3.29), and constructing (4.17) for the semi-direct product case. Introducing monodromies in the groups elements, these actions can be written as chiral WZW models (4.23) and (3.37). When considering Chern-Simons theories on manifolds with non contractible cycles, it has been shown in [8] that the term proportional to $b_{0}$ in (3.29) arises in the associated WZW theory. Thus, one should expect these models to originate from the Chern-Simons formulation of gravity after solving the constraints inside the action once holonomies are properly taken into account. More precisely, this would be the case when adopting the new boundary conditions that have been proposed recently in the context of three dimensional gravity [54], [55] and that give rise to loop groups as asymptotic symmetry groups: for $\mathrm{AdS}_{3}$ gravity the charge algebra yields two copies of $\widehat{\mathrm{SL}(2, \mathbb{R})}$, while in the case of vanishing cosmological constant, it is the centrally extended Poincaré loop group $\operatorname{SL}(2, \widehat{\mathbb{R}) \ltimes \mathfrak{s l}}(2, \mathbb{R})$ that appears. In this sense, actions (3.29) and (4.17) are the $(1+1)$-models representing these boundary degrees of freedom.

In the case of Brown-Henneaux boundary conditions, the asymptotic symmetry group is given by two copies of the diffeomorphism group of the circle and the dual dynamics is controlled by the difference of two $\operatorname{Diff}\left(S^{1}\right)$ invariant actions (3.49). For asymptotically flat spacetimes, similar boundary conditions lead to a boundary dynamics that is controlled by the $\mathrm{BMS}_{3}$ invariant model (4.26). In both cases, once we remove the representative term, we obtain the difference of two chiral bosons (3.52) for $\mathrm{AdS}_{3}$ boundary conditions, and (4.31) in the case of flat geometries. These results are consistent with the earlier derivations [37, 38]. Note however that the periodicity of the chiral fields is determined by the value of the representative(s).

The results of this paper can readily be generalized to other situations that arise in the context of three-dimensional gravity: one could for instance use the general formula (4.13) to work out geometric actions associated to other groups 
with semi-direct product structure like Warped Virasoro [56] or extensions of $\mathrm{BMS}_{3}$ with spin-one generators [57].

Geometric actions can be used to compute one-loop partition functions associated to three-dimensional gravity. It has been shown in [58] for the Virasoro case and for constant representatives that the partition function leads to characters associated with highest weight representations. The key ingredient in this derivation was provided by a transformation that removes the representative from the action. In this paper, we have found a generalization of that transformation for any centrally extended group. Thus, we hope to prove more general connections between characters and partition functions by using relations (3.16) and (3.22).

Another interesting direction corresponds to exploring the connection established between geometric actions and Berry phases [29]. More precisely, it would be interesting to understand the physical content of these phases in the case of systems with $\mathrm{BMS}_{3}$ symmetry or other groups with a semi-direct product structure.

Coadjoint orbits have also appeared recently in the study of the SYK model (see e.g. [59] for a review). In [60, the Hamiltonian associated to a rigid rotation (3.48) in the case where $b_{0}=-\frac{c}{48 \pi}$ is taken as the Euclidean action of the model. In this regard, it would be interesting to understand whether other conserved charges for the Virasoro group, for $\mathrm{BMS}_{3}$ symmetry, or for the Poincaré loop group, could play a similar role.

A most relevant extension of the considerations here consists in modifying from the very beginning the set-up of section 2 by letting $b_{0}=b_{0}(t)$ be a dynamical variable. This means that one no longer considers the dynamics on a fixed coadjoint orbit, but rather a suitable collection of orbits and the associated dynamics. In practice, this can be done for instance by introducing an additional vector $a_{0}=$ $a_{0}(t) \in \mathfrak{g}$. One may then choose the extended pre-symplectic potential

$$
a^{E}=a+\left\langle b_{0}, d a_{0}\right\rangle
$$

and the extended kinetic term

$$
I_{\mathrm{G}}^{E}\left[g, b_{0}, a_{0}\right]=\int_{\gamma^{E}} a^{E} .
$$

The associated pre-symplectic 2 -form is

$$
\Omega^{E}=\Omega+\left\langle d b_{0}, \operatorname{Ad}_{g} \theta\right\rangle+\left\langle b_{0}, d a_{0}\right\rangle .
$$

The extended equations of motion are equivalent to

$$
i_{V} \Omega+\left\langle\dot{b}_{0}, \operatorname{Ad}_{g} i_{V} \theta\right\rangle=0, \quad \dot{a}_{0}=-\operatorname{Ad}_{g} i_{V} \theta, \quad \dot{b}_{0}=0 .
$$

Hence, when choosing the integration constants $b_{0}(t)=\bar{b}_{0}$ to coincide with the constant values of section 2 , the dynamics of the group variables $g$ is unchanged. The 
additional integration constants $\bar{a}_{0}$ are controlled by additional global symmetries that correspond to constant shifts of $a_{0}$.

In particular, in the case of centrally extended groups, we get the kinetic term

$$
I_{\widehat{G}}\left[(g, m),\left(b_{0}, c\right),\left(a_{0}, d\right)\right] .
$$

In this case, the group element $m$ no longer drops out of the problem and the associated global symmetry becomes relevant, and so does the quadratic term $b_{0}^{2}$ in section 3.3.1. Both the orbit representative $b_{0}$ and the central charge $c$ are now dynamical variables. For the orbit representative, this has been analyzed from the geometric actions point of view in the context of "model spaces" in [58, 61] and from the bulk of viewpoint in [62]. For the central extension, it seems that such a generalization has not yet been considered in the context of geometric actions, whereas from the bulk viewpoint it has recently been discussed in [63].

Finally, we will discuss elsewehere the implications of the extended set-up for three-dimensional gravity. In particular, we will study in more detail $(i)$ the bulk duals of the geometric actions obtained here in terms of suitable choices of boundary conditions and by properly taking into account the holonomies and the dynamics of the associated particles, $(i i)$ the interpretation in terms of Goldstone bosons and the connection to non-linear realizations. Most importantly, since the present framework is entirely group-theoretical, there are a priori no obstructions to constructing dynamical actions appropriate to BMS symmetry in four dimensions.

\section{Acknowledgements}

We thank Andrés Gomberoff, Daniel Grumiller, Wout Merbis and Blagoje Oblak for useful discussions. G.B. is grateful to Fondecyt (Chile) Grant Nº1141309 for support during his visit to Chile where part of this work was completed. G.B. is supported by the Fund for Scientific Research-FNRS (Belgium) (convention FRFC PDR T.1025.14 and convention IISN 4.4503.15), H.G. is supported by the Austrian Science Fund (FWF), project P 28751-N2, and P. S-R. is supported by the Fondecyt (Chile) Grant N`3160581.

\section{References}

[1] J. D. Brown and M. Henneaux, "Central Charges in the Canonical Realization of Asymptotic Symmetries: An Example from Three-Dimensional Gravity," Commun. Math. Phys. 104 (1986) 207-226. 
[2] O. Coussaert, M. Henneaux, and P. van Driel, "The Asymptotic dynamics of three-dimensional Einstein gravity with a negative cosmological constant," Class.Quant.Grav. 12 (1995) 2961-2966, arXiv:gr-qc/9506019| [gr-qc].

[3] A. Achucarro and P. Townsend, "A Chern-Simons Action for ThreeDimensional anti-De Sitter Supergravity Theories," Phys.Lett. B180 (1986) 89.

[4] E. Witten, "(2+1)-Dimensional Gravity as an Exactly Soluble System," Nucl. Phys. B311 (1988) 46.

[5] T. Regge and C. Teitelboim, "Role of Surface Integrals in the Hamiltonian Formulation of General Relativity," Ann. Phys. 88 (1974) 286.

[6] E. Witten, "Quantum Field Theory and the Jones Polynomial," Commun.Math.Phys. 121 (1989) 351.

[7] G. W. Moore and N. Seiberg, "Taming the Conformal Zoo," Phys.Lett. B220 (1989) 422.

[8] S. Elitzur, G. W. Moore, A. Schwimmer, and N. Seiberg, "Remarks on the Canonical Quantization of the Chern-Simons-Witten Theory," Nucl.Phys. B326 (1989) 108.

[9] P. Forgacs, A. Wipf, J. Balog, L. Feher, and L. O'Raifeartaigh, "Liouville and Toda Theories as Conformally Reduced WZNW Theories," Phys.Lett. B227 (1989) 214.

[10] M. Bershadsky and H. Ooguri, "Hidden SL(n) Symmetry in Conformal Field Theories," Commun.Math.Phys. 126 (1989) 49.

[11] A. Alekseev and S. L. Shatashvili, "Path Integral Quantization of the Coadjoint Orbits of the Virasoro Group and 2D Gravity," Nucl.Phys. B323 (1989) 719.

[12] K. Skenderis and S. N. Solodukhin, "Quantum effective action from the AdS / CFT correspondence," Phys.Lett. B472 (2000) 316-322, arXiv:hep-th/9910023 [hep-th].

[13] M. Banados, "Three-dimensional quantum geometry and black holes," arXiv:hep-th/9901148 [hep-th].

[14] J. Navarro-Salas and P. Navarro, "Virasoro orbits, AdS(3) quantum gravity and entropy," JHEP 05 (1999) 009, arXiv:hep-th/9903248 [hep-th].

[15] T. Nakatsu, H. Umetsu, and N. Yokoi, "Three-dimensional black holes and Liouville field theory," Prog. Theor. Phys. 102 (1999) 867-896, arXiv:hep-th/9903259 [hep-th]. 
[16] A. Garbarz and M. Leston, "Classification of Boundary Gravitons in $\mathrm{AdS}_{3}$ Gravity," JHEP 05 (2014) 141, arXiv:1403.3367 [hep-th].

[17] G. Barnich and B. Oblak, "Holographic positive energy theorems in three-dimensional gravity," Class. Quant. Grav. 31 (2014) 152001, arXiv:1403.3835 [hep-th].

[18] A. Ashtekar, J. Bicak, and B. G. Schmidt, "Asymptotic structure of symmetry reduced general relativity," Phys.Rev. D55 (1997) 669-686, arXiv:gr-qc/9608042 [gr-qc].

[19] G. Barnich and G. Compere, "Classical central extension for asymptotic symmetries at null infinity in three spacetime dimensions," Class. Quant. Grav. 24 (2007) F15, arXiv:gr-qc/0610130.

[20] G. Barnich and C. Troessaert, "Aspects of the BMS/CFT correspondence," JHEP 05 (2010) 062, arXiv:1001.1541 [hep-th].

[21] G. Barnich and C. Troessaert, "Supertranslations call for superrotations," PoS CNCFG2010 (2010) 010, arXiv:1102.4632 [gr-qc].

[22] G. Barnich, A. Gomberoff, and H. A. González, "Three-dimensional Bondi-Metzner-Sachs invariant two-dimensional field theories as the flat limit of Liouville theory," Phys.Rev. D87 no. 12, (2013) 124032, arXiv:1210.0731 [hep-th].

[23] G. Barnich and B. Oblak, "Notes on the BMS group in three dimensions: I. Induced representations," JHEP 06 (2014) 129, arXiv:1403.5803 [hep-th].

[24] G. Barnich and B. Oblak, "Notes on the BMS group in three dimensions: $\quad$ II. Coadjoint representation," JHEP 03 (2015) 033 , arXiv:1502.00010 [hep-th].

[25] J. Raeymaekers, "Quantization of conical spaces in 3D gravity," JHEP 03 (2015) 060, arXiv:1412.0278 [hep-th].

[26] A. Garbarz and M. Leston, "Quantization of $\mathrm{BMS}_{3}$ orbits: a perturbative approach," Nucl. Phys. B906 (2016) 133-146, arXiv:1507.00339 [hep-th].

[27] A. Campoleoni, H. A. Gonzalez, B. Oblak, and M. Riegler, "BMS Modules in Three Dimensions," Int. J. Mod. Phys. A31 no. 12, (2016) 1650068, arXiv: 1603.03812 [hep-th].

[28] B. Oblak, "BMS Particles in Three Dimensions," arXiv:1610.08526 [hep-th]. 
[29] B. Oblak, "Berry Phases on Virasoro Orbits," arXiv:1703.06142 [hep-th].

[30] B. Kostant, Quantization and Unitary Representations. Springer, 1970.

[31] J.-M. Souriau, Structure des systèmes dynamiques. Dunod, Paris, 1969.

[32] A. A. Kirillov, Elements of the Theory of Representations. Dunod, Paris, 1970.

[33] A. A. Kirillov, Lectures on the orbit method. American Mathematical Society, 2004.

[34] A. Alekseev, L. D. Faddeev, and S. L. Shatashvili, "Quantization of symplectic orbits of compact Lie groups by means of the functional integral," J. Geom. Phys. 5 (1988) 391-406.

[35] L. Guieu and C. Roger, L'Algèbre et le Groupe de Virasoro. Les Publications CRM, Montréal, 2007.

[36] B. Khesin and R. Wendt, The Geometry of Infinite-Dimensional Groups. Springer-Verlag Berlin Heidelberg, 2009.

[37] M. Henneaux, L. Maoz, and A. Schwimmer, "Asymptotic dynamics and asymptotic symmetries of three-dimensional extended AdS supergravity," \begin{tabular}{|l|l|l|}
\hline Annals Phys. 282 (2000) 31-66, arXiv:hep-th/9910013 [hep-th]. \\
\hline
\end{tabular}

[38] G. Barnich and H. A. Gonzalez, "Dual dynamics of three dimensional asymptotically flat Einstein gravity at null infinity," JHEP 05 (2013) 016. arXiv:1303.1075 [hep-th].

[39] B. Rai and V. G. J. Rodgers, "From Coadjoint Orbits to Scale Invariant WZNW Type Actions and 2-D Quantum Gravity Action," Nucl. Phys. B341 (1990) 119-133.

[40] G. W. Delius, P. van Nieuwenhuizen, and V. Rodgers, "The method of coadjoint orbits: an algorithm for the construction of invariant actions," International Journal of Modern Physics A 05 no. 20, (1990) 3943-3983.

[41] H. Aratyn, E. Nissimov, S. Pacheva, and A. H. Zimerman, "Symplectic actions on coadjoint orbits," Phys. Lett. B240 (1990) 127-132.

[42] E. Nissimov and S. Pacheva, "Gauging of geometric actions and integrable hierarchies of KP type," Int. J. Mod. Phys. A16 (2001) 2311-2364, arXiv:hep-th/0009194 [hep-th]. 
[43] P. Salgado-Rebolledo, Symplectic Structure of Constrained Systems: Gribov Ambiguity and Classical Duals for 3D Gravity. PhD thesis, Universidad de Concepción U. \& Université libre de Bruxelles, 2015. http://difusion.ulb.ac.be/vufind/Record/ULB-DIPOT: oai :dipot.ulb.ac.be:2013/220

[44] A. Balachandran, G. Bimonte, K. Gupta, and A. Stern, "Conformal edge currents in Chern-Simons theories," Int.J.Mod.Phys. A7 (1992) 4655-4670, arXiv:hep-th/9110072 [hep-th].

[45] M. Banados, "Global charges in Chern-Simons field theory and the $(2+1)$ black hole," Phys.Rev. D52 (1996) 5816, arXiv:hep-th/9405171|[hep-th].

[46] G. Barnich and G. Compère, "Classical central extension for asymptotic symmetries at null infinity in three spacetime dimensions," Class. Quant. Grav. 24 (2007) F15, gr-qc/0610130. Corrigendum: ibid 24 (2007) 3139.

[47] A. Pressley and G. Segal, Loop Groups. Oxford University Press, 1986.

[48] J. Troost and A. Tsuchiya, "Three-dimensional black hole entropy," JHEP 06 (2003) 029, arXiv:hep-th/0304211 [hep-th].

[49] J. Kim and M. Porrati, "On a Canonical Quantization of 3D Anti de Sitter Pure Gravity," JHEP 10 (2015) 096, arXiv:1508.03638 [hep-th].

[50] J. Balog, L. Feher, and L. Palla, "Coadjoint orbits of the Virasoro algebra and the global Liouville equation," Int. J. Mod. Phys. A13 (1998) 315-362, arXiv:hep-th/9703045 [hep-th].

[51] P. Baguis, "Semidirect products and the Pukanszky condition," Journal of Geometry and Physics. 25 (1998) 245-270.

[52] G. Barnich, L. Donnay, J. Matulich, and R. Troncoso, "Super$\mathrm{BMS}_{3}$ invariant boundary theory from three-dimensional flat supergravity," JHEP 01 (2017) 029, arXiv:1510.08824 [hep-th].

[53] H. A. Gonzalez and M. Pino, "Boundary dynamics of asymptotically flat 3D gravity coupled to higher spin fields," JHEP 05 (2014) 127, arXiv:1403.4898 [hep-th].

[54] D. Grumiller and M. Riegler, "Most general $\mathrm{AdS}_{3}$ boundary conditions," JHEP 10 (2016) 023, arXiv:1608.01308 [hep-th].

[55] D. Grumiller, W. Merbis, and M. Riegler, "Most general flat space boundary conditions in three-dimensional Einstein gravity," arXiv:1704.07419 [hep-th]. 
[56] G. Compère, W. Song, and A. Strominger, "New Boundary Conditions for AdS3," JHEP 05 (2013) 152, arXiv:1303.2662 [hep-th],

[57] S. Detournay and M. Riegler, "Enhanced Asymptotic Symmetry Algebra of 2+1 Dimensional Flat Space," Phys. Rev. D95 no. 4, (2017) 046008, arXiv: 1612.00278 [hep-th].

[58] A. Alekseev and S. L. Shatashvili, "From geometric quantization to conformal field theory," Commun. Math. Phys. 128 (1990) 197-212.

[59] J. Maldacena and D. Stanford, "Remarks on the Sachdev-Ye-Kitaev model," Phys. Rev. D94 no. 10, (2016) 106002, arXiv:1604.07818 [hep-th].

[60] D. Stanford and E. Witten, "Fermionic Localization of the Schwarzian Theory," arXiv:1703.04612 [hep-th].

[61] H. La, P. Nelson, and A. S. Schwarz, "Virasoro model space," Communications in Mathematical Physics 134 no. 3, (12, 1990) 539-554. http://dx.doi.org/10.1007/BF02098446.

[62] G. Compère, P. Mao, A. Seraj, and M. M. Sheikh-Jabbari, "Symplectic and Killing symmetries of $\mathrm{AdS}_{3}$ gravity: holographic vs boundary gravitons," JHEP 01 (2016) 080, arXiv:1511.06079 [hep-th].

[63] C. Bunster and A. Pérez, "Superselection rule for the cosmological constant in three-dimensional spacetime," Phys. Rev. D91 no. 2, (2015) 024029, arXiv:1412.1492 [hep-th]. 